\title{
Evaluation the efficacy and side effects of postoperative analgesia in patients after orthopedic surgery
}

${ }^{1}$ Konkayev A., ${ }^{1}$ Yeltayeva A., ${ }^{2}$ Sainov M.

${ }^{1}$ Astana Medical University, ${ }^{2}$ Institution of

Traumatology and Orthopedia

Astana, Kazakhstan 
Background and Goal of Study: Orthopedic surgery in adults needs an optimal postoperative pain management. In case of inadequate pain relief in $20 \%$ of patients after surgery may develop chronic pain (1). The aim of study was to evaluate the efficacy and side effects of postoperative analgesia in patients after orthopedic surgery. 
Materials and methods: After obtaining written informed consent and local ethic committee approval 228 adults (aging 43.5 \pm 16.5 , ASA I-II) scheduled for orthopedic surgery. Numeric rating scale (NRS), efficacy and side effects score (Kongsberg satisfaction score KSS) and mobility degree will be registered every hour during the first 8 hours postoperatively (1). Postoperative pain was treated with doses of $100 \mathrm{mg}$ tramadol i.m. on demand. Data were processed unpaired t-test with Statistica 6.0 (StatSoft Inc., Tulsa, OK, USA); $p<0.05$ significant. Data are means $\pm S D$. 
Results and Discussion: Mean KSS was $18 \pm 2$ in the first hour after surgery. During the next 7 hours, it remained between 19 and 24 corresponding to good satisfaction. A slowly increasing degree of mobilization was revealed during the first 8 postoperative hours. By analyzing different types of anaesthesia for knee replacement, we found a higher KSS during the first 4 hours postoperatively $(p<0.05)$ after spinal anaesthesia as compared with total intravenous anaesthesia. 
Results and Discussion: NRS in second hour after spinal anesthesia $(3.1 \pm 0.4)$ was lower than in patients after intravenous anaesthesia $(5.9 \pm 0.9, p<0.05)$. Total analgetic consumption (tramadol) was higher in patients after general anaesthesia $(50.1 \pm 5.8 \mathrm{mg}$ vs $14.3 \pm 3.1 ; p<0.02)$ on the first day after operation.

Conclusion(s): Using KSS may be a useful for monitoring the status, efficacy and safety of postoperative pain control of orthopedic patients.

References: (1) Kuklin V., Skraastad E., Ernst G. et al. Kongsberg satisfaction score: a novel system for monitoring the status, efficacy and safety of the postoperative pain treatment. International Anaesthesia Research Society Annual Meeting, San Diego, California, USA, May 4-7, 2013. 